\title{
Family size solar dryer for an estimation of the heat transfer coefficient
}

\author{
T. M. Jaballa \\ Mechanical Engineering Department, \\ College of Engineering, El-Fateh University, Tripoli, Libya
}

\begin{abstract}
Knowledge of the heat transfer coefficient is believed to be of great contribution in the course of a full design of a solar dryer. To find an expression for the heat transfer coefficient, the general logarithmic velocity profile for turbulent flow is used for the analysis. It is assumed that the fluid is air at atmospheric pressure, and that the thin wall laminar sublayer has a small or negligible effect on the value of the fluid bulk temperature. The final form of the correlation equation gives a relation between Nusselt number and Reynolds' number, and is given in a form which is easy to remember by the solar dryer designers. Comparison of the predicted values indicates that the correlation can predict values to within $\pm 6 \%$ of experimental values reported in literature. Expected errors of nearly $\pm 25 \%$ were observed when the correlation predictions compared with well known correlations found in literature and in text books.

Keywords: heat transfer coefficient, crops solar dryers, turbulent velocity profile, turbulent thermal diffusivity.
\end{abstract}

\section{Introduction}

Drying crops such as hay, corn, beans, tomatoes, onions, etc., has been known to man for thousands of years. The drying method used then was purely natural; by simply distribute the crop on a flat ground, and let nature dry it. Very little of industrial pollutants those days were existed, and the insects effect was not of any concern. Today this same method is still used locally, in the presence of industrial and growing natural pollution. Dates, tomatoes, unions, apricots, and even meat and fish are dried in open areas. Many of the diseases in the African countries are related to foods dried this way. A cleaner and a more healthy 
method is required, especially when drying products that can get dirty due to adhesion and sticking of exhaust gases, ashes, and dust, or when drying meats and some fruits which contain sweet juices, and aromas that attract insects.

A proposed program to fully design a family solar dryer to dry local crops is considered by the Mechanical Engineering Department, El-Fateh University. The proposal consists of three phases. The first is to make a preliminary design of a simple and cheap dryer geometry and to report the moisture content of all the crops to be dried. The second phase is the thermal design and analysis, a purely theoretical study. The third phase is the design and manufacturing of a cheap type solar dryer and the measurements of all the parameters needed for the analysis. In this paper only one task is presented, the derivation of the heat transfer coefficient correlation that is simple enough to be remembered by the solar dryer designers.

\section{Crops solar dryers}

Crops solar dryers can take different geometries and sizes. The airflow in solar dryers can be a natural flow or a convective one. The most widely used solar dryer of the medium size is the tunnel type solar dryer, figure 1 [1]. It is used to dry products by warm air. The energy needed for this type is made available by either trapping the solar energy using a plastic cover or by sing a flat plate solar collector as a cover.

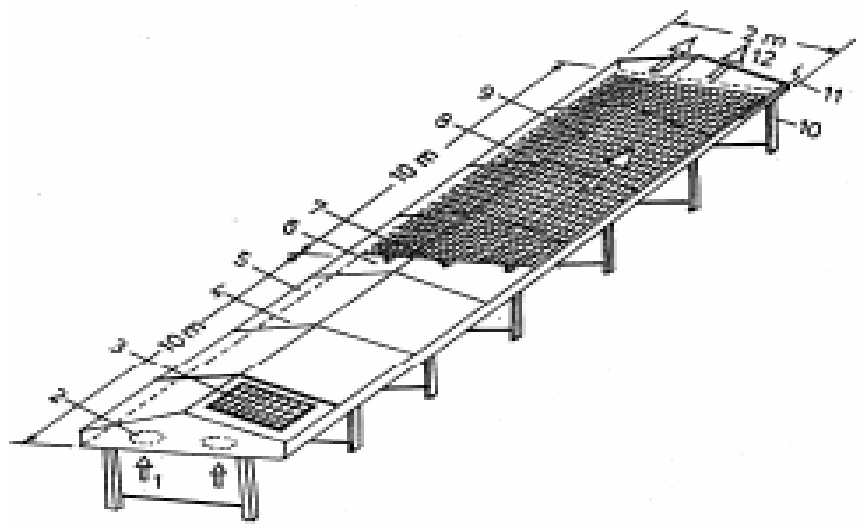

Figure 1: $\quad$ Tunnel type solar dryer [1].

The box type solar dryer is another widely used type. The box sides and top are covered by transparent plastic sheet to trap the solar energy while its bottom is well insulated. It contains one or more perforated shelves to contain the product to be dried. Figure 2 shows an example of box type solar dryers [2]. Other types of solar dryers can be considered to have both advantages of the box solar dryer and the tunnel type solar dryer as in figure 3 [3]. It can be seen that a flat plate solar collector is also needed in this type. The collector plate can be tilted or rotated to face the sun. 


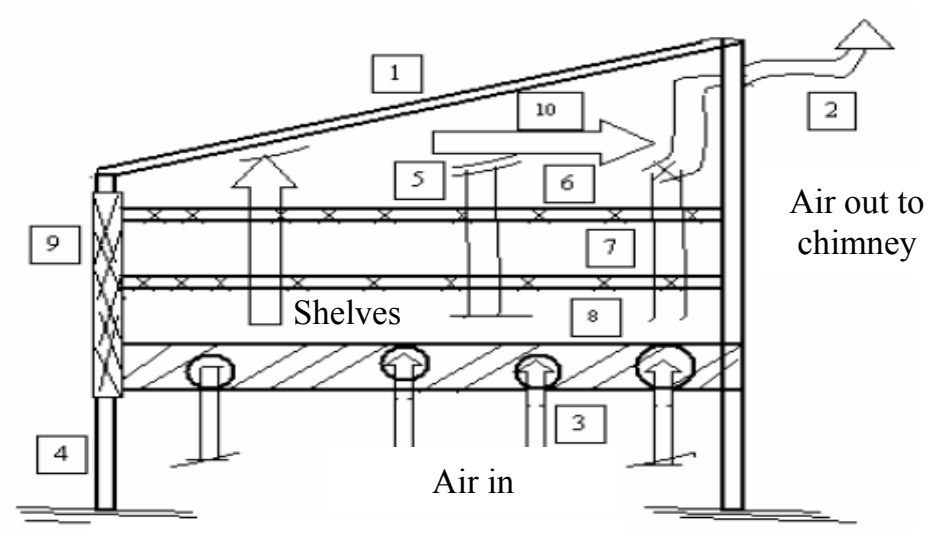

Figure 2: $\quad$ Box type solar dryer [2].

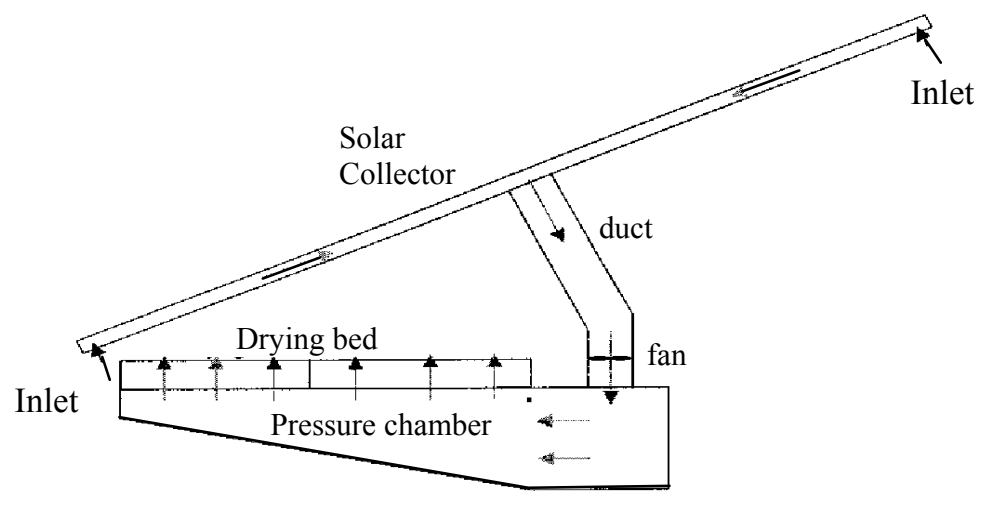

Figure 3: $\quad$ Convective crop solar dryer [3].

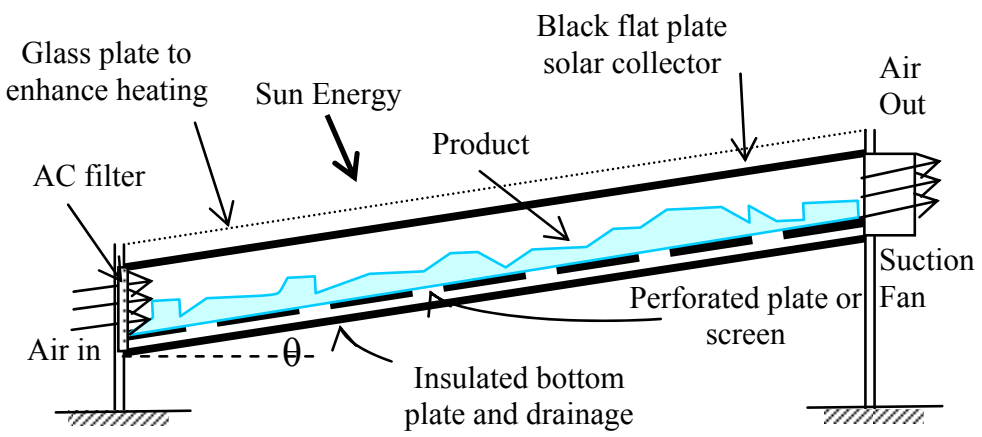

Figure 4: $\quad$ The proposed solar dryer. 
The solar collector plate can be the bottom of the dryer channel provided that the cannel aspect ratio is large and only air flows through it. It also can be on the top of the dryer channel if the products are to be placed in the channel. It $s$ believed that in this case the channel can be considered of large aspect ratio due to the small thickness of the space between the top hot plate collector and the edge of the product, which is the case to be considered in this paper.

Figure 4 shows a proposal for a horizontal or slightly inclined simple and cheap solar dryer. This dryer must have its bottom and sides well insulated. Forced convection is guaranteed by forced flow of air under the collector plate. The air stream is heated due to direct contact with the plate above it. The products inside the duct work as a trip to force the flow to be turbulent flow even when the flow velocity is low.

\section{The heat transfer coefficient}

The heat transfer mechanism to the air from the upper hot plate depends on the characteristics of the turbulent velocity. The velocity profile is known to follow the law of the wall as seen in figure 5.

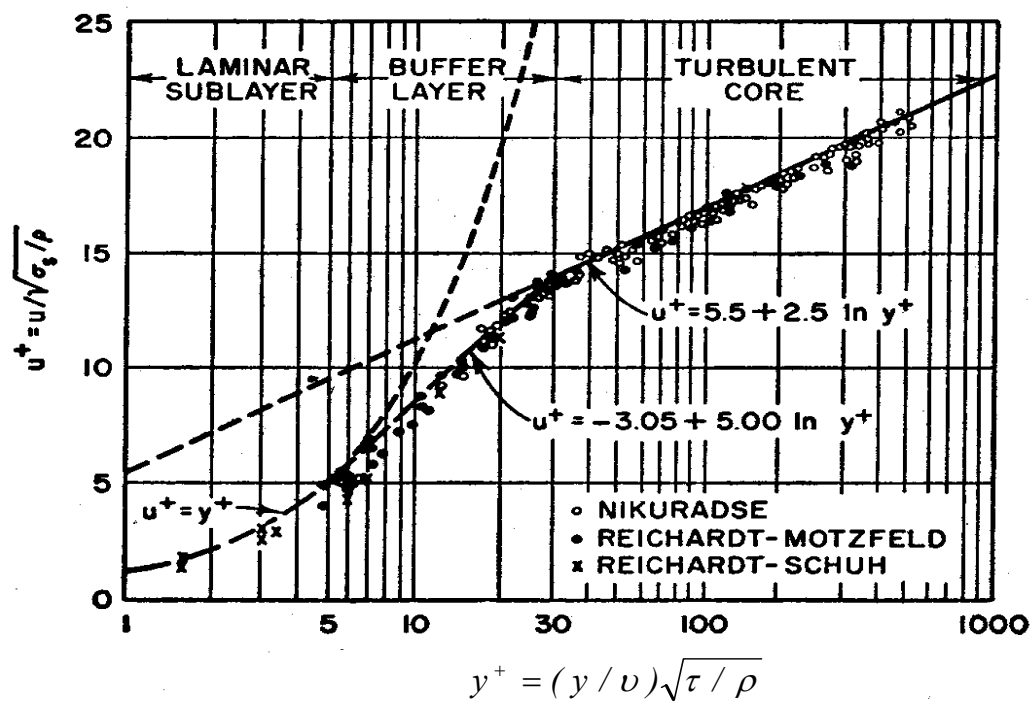

Figure 5: Velocity distribution in turbulent flow [4].

Giedt [4] gave the profiles of temperature for the three regions according to the law of the wall:

Laminar sublayer:

$$
T_{w}-T=\frac{q_{w} P r}{\rho c_{p} \sqrt{\tau_{w} / \rho}} y^{+}, \quad 0<\mathrm{y}^{+}<5 .
$$


Buffer layer:

$$
T_{1}-T=\frac{5 q_{w} \ln \left[1+E \operatorname{Pr}\left(\left(y^{+} / 5\right)-1\right)\right]}{E \rho c_{p} \sqrt{\tau_{w} / \rho}}, \quad 5<\mathrm{y}<30 .
$$

Turbulent core:

$$
T_{2}-T=\frac{2.5 q_{w}}{E \rho c_{p} \sqrt{\tau_{w} / \rho}} \ln \frac{y}{y_{2}}, \quad y_{2}=\left.y\right|_{y_{2}^{+}=30}, \quad \mathrm{y}^{+}>30 .
$$

In the above equations, $\mathrm{T}_{\mathrm{w}}$ is the wall temperature, $\mathrm{T}_{1}=\mathrm{T}\left(\mathrm{y}^{+}=5\right), \mathrm{T}_{2}=\mathrm{T}\left(\mathrm{y}^{+}=30\right)$ as shown in figure 2, $\mathrm{E}$ in the second equation is the ratio of the eddy diffusivity of heat to that of momentum, $\epsilon_{H} / \epsilon_{M}$ and $\mathrm{y}$ in the third equation is the distance from the hot plate to a point in the turbulent core. Although $\mathrm{E}$ was found to be nearly 1.4 , many analyses use this ratio as one [4].

In order to find the bulk temperature, $T_{B}$, at a section, the above equations need to be integrated, and the Nusselt number must be defined using $T_{B}$. To simplify the analysis, the turbulent temperature profile, as shown in figure 6 , is considered to be almost uniform at a section except at the laminar sublayer.

Substituting $\mathrm{y}^{+}=5$ in equation (1), and $\mathrm{y}^{+}=30$ in equation (2), yields the following:

$$
\begin{aligned}
\Delta T_{w}=T_{w}-T_{1}= & \frac{5 \times \operatorname{Pr} \times q_{w}}{\rho c_{p} \sqrt{\tau_{w} / \rho}}, \\
\Delta T_{\text {buffer }} & =T_{1}-T_{2}=\frac{5 q_{w}}{E \rho c_{p} \sqrt{\tau_{w} / \rho}} \ln [1+5 E \operatorname{Pr}],
\end{aligned}
$$

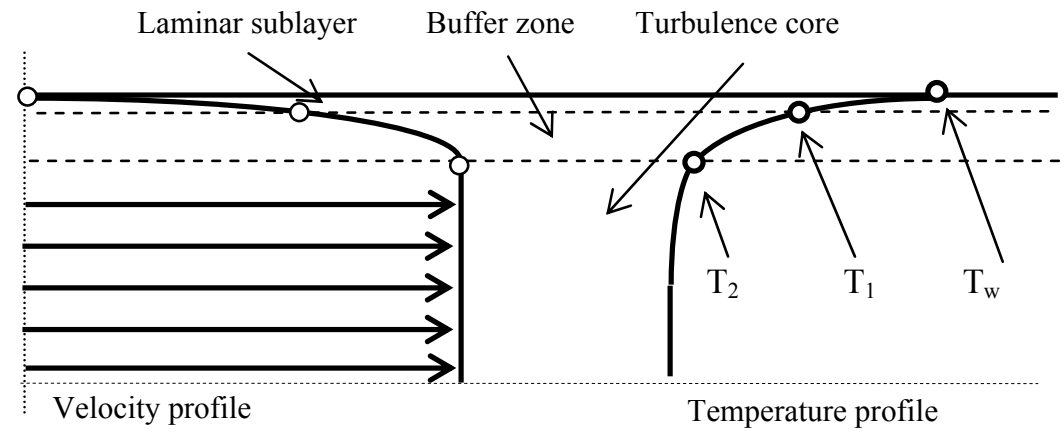

Figure 6: Velocity and temperature profiles under the dryer hot plate.

Where:

$\Delta T_{w}=$ the increase or decrease in temperature through the laminar sublayer, $\Delta T_{\text {buffer }}=$ the increase or decrease in temperature through the buffer layer. 


\section{Determination of the channel bulk temperature}

The fluid bulk temperature is found by choosing a control volume at the outer edge of the buffer zone, figure 7, assuming that the laminar sublayer and the buffer zone are very small compared to the height of the channel, and that all the heat they receive from the upper wall is transferred to the bulk of the fluid in the turbulent zone by conduction only.

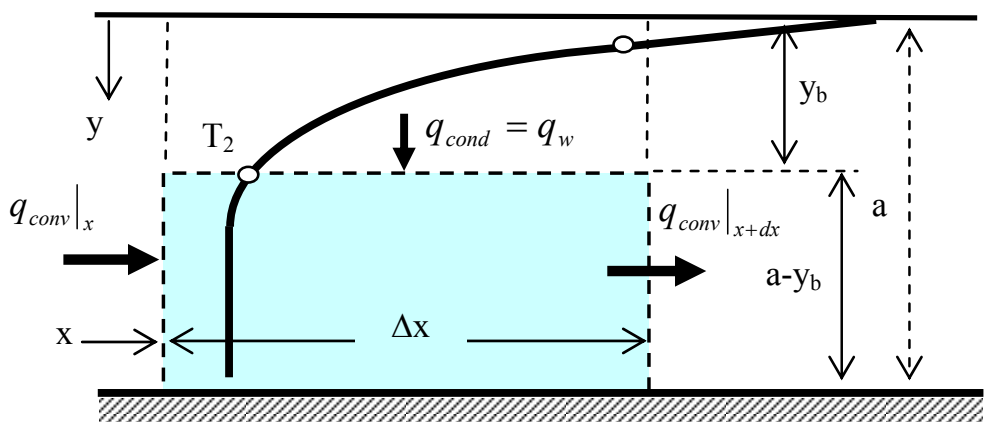

Figure 7: The control volume for the heat balance analysis.

Assuming that the lower bottom of channel is completely insulated, and that the heat transfer to the fluid in the turbulent core is from the upper wall, the heat balance is given by:

$$
\begin{gathered}
q_{\text {cond }}+\left.q_{\text {conv }}\right|_{x}=\left.q_{\text {conv }}\right|_{x+d x}, \\
q_{w} \cdot w \cdot \Delta x=\rho u_{m} \cdot w \cdot\left(a-y_{b}\right) \frac{d h}{d x} d x=\rho u_{m} \cdot w \cdot\left(a-y_{b}\right) \cdot c p \frac{d T}{d x} \cdot \Delta x, \\
q_{w}=\rho u_{m} \cdot\left(a-y_{b}\right) \cdot c_{p} \frac{d T}{d x} .
\end{gathered}
$$

Choosing a more general control volume in the turbulent core at a distance $\mathrm{y}>\mathrm{y}_{\mathrm{b}}$ from the wall, noting that in this region $q_{\text {cond }}=-\rho c_{p} \in_{H}(d T / d y)$ :

$$
\begin{gathered}
-\rho c_{p} \in_{H} \frac{d T}{d y} \cdot w \cdot \Delta x=\rho u_{m} \cdot w \cdot(a-y) \cdot c_{p} \frac{d T}{d x} \cdot \Delta x, \\
-\in_{H} \frac{d T}{d y}=u_{m}(a-y) \frac{d T}{d x} .
\end{gathered}
$$

Dividing equation (6) by equation (7), arranging and integrating from $\mathrm{T}_{2}$ at $\mathrm{y}_{\mathrm{b}}$ to $\mathrm{T}$ at $\mathrm{y}$, we get:

$$
T-T_{2}=-\frac{q_{w}}{\rho c_{p} \in_{H}\left(a-y_{b}\right)}\left[a\left(y-y_{b}\right)+0.5\left(y_{b}^{2}-y^{2}\right)\right]
$$


The above equation is used to determine the equation for the fluid bulk temperature, which in turn used to find the equation for the Nusselt number. The fluid bulk temperature is defined as [8]:

$$
T_{B}=\frac{\int_{y_{b}}^{a} \rho c_{p} T u_{m} w d y}{\int_{y_{b}}^{a} \rho c_{p} u_{m} w d y}=\frac{1}{a-y_{b}} \int_{y_{b}}^{a} T d y .
$$

where $\mathrm{u}_{\mathrm{m}}$ is assumed uniform in the turbulent region, and $\rho$, and $c_{p}$ are considered constants. Substitution for T from eqn. (8) in eqn. (9) gives:

$$
T_{2}-T_{B}=\frac{q_{w}}{\rho c_{p}\left(a-y_{b}\right)^{2} \in_{H}}\left[\frac{1}{3}\left(a^{3}-y_{b}^{3}\right)+a y_{b}^{2}-a^{2} y_{b}\right] .
$$

Simple division of the term between the square brackets by $\left(a-y_{b}\right)^{2}$ reduces the above equation to the following:

$$
T_{2}-T_{B}=\frac{q_{w}}{\rho c_{p} \in_{H}}\left[\frac{1}{3} a-y_{b}\right] \text {. }
$$

Given that $T_{W}-T_{B}=\left(T_{w}-T_{1}\right)+\left(T_{1}-T_{2}\right)+\left(T_{2}-T_{B}\right)$, equations (4), and (5) are then used to find the equation for $\left(\mathrm{T}_{\mathrm{w}}-\mathrm{T}_{\mathrm{B}}\right)$ as follows:

$$
\begin{aligned}
T_{W}-T_{B}=\frac{5 \times \operatorname{Pr} \times q_{w}}{\rho c_{p} \sqrt{\tau_{w} / \rho}}+\frac{5 q_{w}}{\rho c_{p} \sqrt{\tau_{w} / \rho}} & \ln [1+5 \operatorname{Pr}] \\
& +\frac{q_{w}}{\rho c_{p} \in_{H}}\left[\frac{1}{3} a-y_{b}\right]
\end{aligned}
$$

Substitution of $y_{b}=\frac{30 v}{\sqrt{\tau_{w} / \rho}}$ and $a=\frac{y_{a}^{+} v}{\sqrt{\tau_{w} / \rho}}$ into equation (11) and rearranging, gives:

$$
T_{W}-T_{B}=\frac{q_{w}}{\rho c_{p}} \frac{1}{\sqrt{\tau_{w} / \rho}}\left[\begin{array}{c}
5 \operatorname{Pr}+5 \ln [1+5 \operatorname{Pr}] \\
+\frac{v}{\epsilon_{H}}\left[\frac{1}{3} y_{a}^{+}-30\right]
\end{array}\right] .
$$

The turbulent heat diffusivity relation is not given very explicitly in many of the literature papers that have been reviewed. A relation for this quantity as a function of Reynolds' number can be arrived at from reference [6] as:

$$
\frac{v}{\epsilon_{H}}=2547.57 R e^{-1.121} .
$$

The above equation is found to take the exponential form as in figure 8 . 


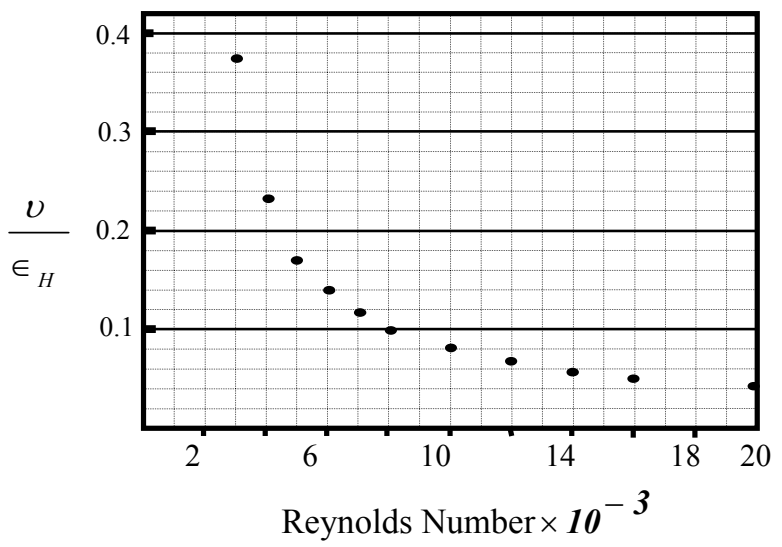

Figure 8: The dependence of the ratio $v / \in_{H}$ on the air flow Reynolds number.

In the above equation Reynolds' number is defined as: $R e=V D_{H} / v$ and is in the range of $10^{4}$ to $5 \times 10^{4}$. It should be mentioned that the results of eqn. (13) are not verified to be correct over a wide range of Re yet, and the equation should be used with great caution. Since the aspect ratio of channel dryers is high, $w>a$, it follows that the hydraulic diameter is:

$$
D_{H}=\frac{4 A}{P}=\frac{4(a \times w)}{2(a+w)} \cong \frac{2(a \times w)}{w}=2 a .
$$

Inserting equation 13 into equation (12), we get:

$$
T_{W}-T_{B}=\frac{q_{w}}{\rho c_{p}} \frac{1}{\sqrt{\tau_{w} / \rho}}\left[\begin{array}{c}
5 \operatorname{Pr}+5 \ln [1+5 \operatorname{Pr}] \\
+\frac{2547.57}{\operatorname{Re} e^{1.121}}\left[\frac{1}{3} y_{a}^{+}-30\right]
\end{array}\right] .
$$

where;

$$
y_{a}^{+}=\frac{a \sqrt{\tau_{w} / \rho}}{v}=\frac{a \cdot u_{m} \sqrt{f / 8}}{v}=\frac{1}{2} \sqrt{f / 8} \operatorname{Re} .
$$

Using $\sqrt{f / 8}=0.191918 R e^{-0.1225}$ for the operating range of $\mathrm{Re}=4 \times 10^{3}$ to $\operatorname{Re}=10^{5}$, and $\sqrt{\tau_{w} / \rho}=u_{m} \sqrt{f / 8}$ from the balance of the shear forces and the pressure forces [5], in eqn. (14) we get: 


$$
T_{W}-T_{B}=\frac{q_{w}}{\rho c_{p} u_{m}}\left[\begin{array}{ll}
\frac{(5 \operatorname{Pr}+5 \ln [1+5 \operatorname{Pr}])}{\left(0.191918 R e^{-0.1225}\right)} \\
+424.5949 R e^{-0.121}-398243 & R e^{-0.9985}
\end{array}\right] .
$$

The above equation is the general equation to predict the temperature difference between the wall and the fluid bulk temperature.

For air at the average working temperatures in dryers, Prandtl number is very close to 0.69 . Inserting this into the above equation we get:

$$
T_{W}-T_{B}=\frac{q_{w}}{\rho c_{p} u_{m}}\left[\begin{array}{l}
56.87 R e^{0.1225} \\
+424.5949 R e^{-0.121}-398243 R e^{-0.9985}
\end{array}\right] .
$$

\section{The heat transfer correlation}

By definition, Stanton number is given as:

$$
S t=\frac{N u}{\operatorname{Pr} \cdot \operatorname{Re}}=\frac{h}{\rho c_{P} u_{m}}=\frac{q_{w}}{\rho c_{P} u_{m}\left(T_{w}-T_{B}\right)} .
$$

Substituting for $\left(T_{w}-T_{B}\right)$, and for $\operatorname{Pr}=0.69$ in the above equation, and using the least square method to take the form $a R e^{n}$, we get:

$$
N u=0.0086 \cdot R e^{0.86}=0.86 \cdot R e^{0.86} / 100
$$

Equation (15) is the final form of the correlation between Nusselt number and Reynolds' number. It can be seen that this relation is easy to remember, and that the only numeric value to be memorized for both the constant and the index is 0.86 . The author will point to this equation as the Giedt correlation after the late Prof. Warren A Giedt whose contribution in thermophysics and heat transfer is outstanding.

\section{Comparison of the correlation predictions}

Giedt's correlation, eqn. (15), must be validated using experimental measurements and other available correlations found in literature.

\subsection{Comparison with experimental measurements}

Since the correlation is derived using turbulent assumptions, the results of Nusselt numbers are compared with experimental results made by [7] for the range of $10^{4}<\operatorname{Re}<5 \times 10^{4}$. Predictions out of this range of Re could not be verified.

Table 1 shows the correlation predictions of $\mathrm{Nu}$, and surprisingly it predicted the values of Nusselt numbers to within $\pm 5 \%$ of the experimental values. 
Table 1: Comparison of the predicted $\mathrm{Nu}$ of Giedt's correlation with experimental results of [7].

\begin{tabular}{|l|l|l|l|l|l|l|l|}
\hline Re & 5000 & 10000 & 20000 & 30000 & 40000 & 50000 & 60000 \\
\hline Giedt's eqn. & 13.05 & 23.7 & 43. & 60.9 & 77.5 & 94.5 & 110.6 \\
\hline Experiments & - & 24.87 & 43.31 & 59.9 & 75.4 & 90.14 & - \\
\hline Error, \% & - & -4.7 & -0.7 & +1.6 & +2.8 & +4.8 & - \\
\hline
\end{tabular}

\subsection{Comparison with some correlations in literature}

In order to validate the predictions of Giedt's correlation, it is compared with some correlations found in literature. It was not clear from these correlations however that the circumstances are the same.

\subsubsection{Reynolds analogy}

The definition of Stanton number from Reynolds analogy is given by:

$$
S t=\frac{\mid N u}{\operatorname{RePr}}=\frac{h}{\rho c_{P} u_{m}}=\frac{f}{2}=\frac{2 \tau_{w}}{2 \rho u_{m}^{2}}=\frac{\tau_{w}}{\rho u_{m}^{2}} .
$$

Substitution of the definition of turbulent shear, $\tau_{w}=\mathbf{0 . 0 3 8 6} \rho u_{m}^{2} R e^{-1 / 4}$ [5], and $\operatorname{Pr}=\mathbf{0 . 6 9}$ into the above equation yields:

$$
N u=0.0266 R e^{3 / 4}
$$

Table 2: $\quad$ Comparison of the predicted $\mathrm{Nu}$ with eqn (16).

\begin{tabular}{|l|c|c|c|c|c|c|c|}
\hline $\mathrm{Re}$ & 5000 & 10000 & 20000 & 30000 & 40000 & 50000 & 60000 \\
\hline Giedt's eqn. & 13.05 & 23.7 & 43. & 60.9 & 77.5 & 94.5 & 110.6 \\
\hline Eqn. (16) & 15.81 & 26.6 & 44.7 & 60.63 & 75.2 & 89.0 & 102 \\
\hline Error, \% & 18 & 12 & 5 & 0.6 & -3 & -5.6 & -7.7 \\
\hline
\end{tabular}

\subsection{Colburn analogy}

Colburn analogy modifies equation (16) by the factor of $\operatorname{Pr}^{1 / 3}$ as follows,

$$
N u=0.0266 \operatorname{Re}^{3 / 4} \operatorname{Pr}^{1 / 3} \text {. }
$$

Table 3: $\quad$ Comparison of the predicted $\mathrm{Nu}$ with eqn. (17).

\begin{tabular}{|l|c|c|c|c|c|c|c|}
\hline $\mathrm{Re}$ & 5000 & 10000 & 20000 & 30000 & 40000 & 50000 & 60000 \\
\hline Giedt's eqn. & 13.05 & 23.7 & 43. & 60.9 & 77.5 & 94.5 & 110.6 \\
\hline Eqn. (17) & 14 & 23.5 & 39.5 & 53.6 & 66.5 & 79. & 90. \\
\hline Error, \% & 8.1 & 0.2 & -7.8 & -12.5 & -15.2 & -19.0 & -23 \\
\hline
\end{tabular}




\subsubsection{Air flow in smooth ducts}

Holman [9] gave the following correlation for air flow in smooth ducts:

$$
N u_{d}=0.023 \operatorname{Re}^{0.8} \operatorname{Pr}^{0.4} \text {, }
$$

Table 4: $\quad$ Comparison of the predicted $\mathrm{Nu}$ of Giedt correlation with eqn. (18).

\begin{tabular}{|l|c|c|c|c|c|c|c|}
\hline Re & 5000 & 10000 & 20000 & 30000 & 40000 & 50000 & 60000 \\
\hline Giedt's eqn. & 13.05 & 23.7 & 43. & 60.9 & 77.5 & 94.5 & 110.6 \\
\hline Eqn. (18) & 18.04 & 31.4 & 54.7 & 75.6 & 95 & 113.9 & 131.8 \\
\hline Error, \% & 27 & 25 & 21 & 19 & 19 & 17 & 16 \\
\hline
\end{tabular}

6.3.2 Flows of fluids with $0.5<P r<1.0$

Karlekar and Desmond [5] reported that for flows in smooth pipes, the following correlation applies:

$$
N u=0.022 \operatorname{Re}^{0.8} \operatorname{Pr}^{0.6},
$$

Table 5: $\quad$ Comparison of the predicted $\mathrm{Nu}$ with equation (19).

\begin{tabular}{|l|l|l|l|l|l|l|l|}
\hline Re & 5000 & 10000 & 20000 & 30000 & 40000 & 50000 & 60000 \\
\hline Giedt's cor. & 13.05 & 23.7 & 43. & 60.9 & 77.5 & 94.5 & 110.6 \\
\hline Eqn. (19) & 16. & 27.9 & 48.6 & 67.2 & 84.6 & 101. & 117. \\
\hline Error, \% & 18.4 & 15. & 11.5 & 9.4 & 8.4 & 6.4 & 5.4 \\
\hline
\end{tabular}

\section{Discussions and conclusions}

From the above comparisons, it can be seen that the developed correlation is strikingly in good agreement with the experimental measurements of [7]. The $5 \%$ error in almost all engineering applications, especially in the field of convection heat transfer, is considered very acceptable.

Comparison with other correlations shows higher percentage errors. Holman, [6], reported that equation (18) predicts values within $\pm 25 \%$. The predicted values of Giedt correlation are shown to be within this range of errors when compared with almost all correlations used for comparison. Except for eqn (18), Giedt's correlation predictions are found to be within $20 \%$ of those predicted by other correlations in the range of $10000<\operatorname{Re}<50000$.

It can be concluded that the Giedt correlation can be used with confidence for the purpose of calculating the heat transfer coefficient within errors of nearly $5 \%$. It has a simple and easy to remember form. T he correlation can be used for comparison when conducting experimental measurements in forced convection solar dryers, which will be conducted in a later phase of the design of family size solar dryer. 


\section{Nomenclature}
A
solar collector flow area, $\mathrm{m}^{2}$
a
distance between top and bottom plates, $\mathrm{m}$
E
the ratio $\epsilon_{H} / \epsilon_{M}$
the friction coefficient
$\mathrm{h}$
the heat transfer coefficient, $\mathrm{W} / \mathrm{m}^{2} \mathrm{~K}$
$\mathrm{k}$
the conductivity, $\mathrm{W} / \mathrm{mK}$
$\mathrm{q}_{\mathrm{w}}$
the wall heat transfer, $\mathrm{W} / \mathrm{m}^{2}$
$\mathrm{T}$
temperature, $\mathrm{K}$
$\mathrm{T}_{\mathrm{w}}$
wall temperature, $\mathrm{K}$
$\mathrm{T}_{1}$
temperature of the edge of the laminar sublayer, $\mathrm{K}$
$\mathrm{T}_{2}$
temperature of the edge of the buffer zone, $\mathrm{K}$
$\mathrm{u}$
air velocity, $\mathrm{m} / \mathrm{s}$
$\mathrm{u}^{+}$
non-dimensional velocity
$\mathrm{X}$
coordinate in the flow direction, $\mathrm{m}$
$\mathrm{y}$
coordinate and distance from the dryer collector plate, $\mathrm{m}$
$\mathrm{y}^{+}$
non- dimensional coordinate
$\mathrm{y}_{\mathrm{b}}$
distance from collector top plate to the outer edge of the buffer zone
$\epsilon_{H}$
turbulent heat diffusivity
$\epsilon_{M}$
turbulent momentum diffusivity
$\rho$
density, $\mathrm{kg} / \mathrm{m}^{3}$
$v$
kinematic viscosity, $\mathrm{m}^{2} / \mathrm{s}$
$\tau$
(also $\sigma_{s}$ ) shear stress, $\mathrm{N} / \mathrm{m}^{2}$
$\tau_{w}$
wall shear stress, $\mathrm{N} / \mathrm{m}^{2}$

$f$

\section{References}

[1] B. K. Bala, and Sem Janjai, 'Solar drying of fruits, vegetables, spices, medicinal plants and fish: Developments and Potentials, International Solar Food Processing conference, 2009, p. 1. 
[2] B. A. Ezekoye, and O. M. Enabe, 'Development and Performance Evaluation of Modified Integrated Passive Solar Grain Dryer', The Pacific Journal of Science and Technology, Volume 7, No 2, November, 2006, p. 185

[3] Soren O. Jensen, and Erik F. Kristensen, 'Test of a solar crop dryer', Solar Energy Center and Danish Technological Institute, Jan 2001, p. 4

[4] Prof. Warren H. Giedt, 'Principles of Engineering Heat Transfer', Second Edition, 1967, p 177-180

[5] Karlekar, B. V. and Desmond, R. M., 'Heat Transfer', Second Edition, 1982

[6] M. A. S. Malik, F. H. Buelow, 'Analytical Model for Determinating the Heat Transfer Coefficients in No-Glass Flat Plate Collectors Used for Drying Agricultural Crops', Sun, Mankind's Future Source of Energy, Volume 3, Pergamon, 1978, p 1946.

[7] H. M. Tan, W. W. S. Charters, 'An Experimental Investigation of Forced convection Heat Transfer for Fully Developed Turbulent Flow in a rectangular Duct with Asymmetric Heating', journal of Solar Energy, V13, p121, 1970.

[8] Fox, R. W. and McDonald, A. T., 'Introduction to Fluid Mechanics', Fourth Edition, John Wiley \& Sons, Inc., 1994.

[9] Holman, J. P., 'Heat Transfer', Fifth Edition, McGraw-Hill, 1981, p. 227 